\title{
An Agent-Based Infectious Disease Model of Rubella Outbreaks
}

\author{
Setsuya Kurahashi
}

\begin{abstract}
This study proposes a simulation model of rubella. SIR (Susceptible, Infected, Recovered) model has been widely used to analyse infectious diseases such as influenza, smallpox, bioterrorism, to name a few. On the other hand, agentbased model begins to spread in recent years. The model enables to represent the behaviour of each person on the computer. It also reveals the spread of infection by simulation of the contact process among people in the model. The study designs a model based on smallpox and Ebola fever model in which several health policies are decided such as vaccination, the gender-specific workplace and so on. The infectious simulation of rubella, which has not yet vaccinated completely for men in Japan, is implemented in the model. As results of experiments using the model, it has been found that preventive vaccine to all the men is crucial factors to prevent the spread in women.
\end{abstract}

Keywords Agent-based model $\cdot$ Infectious disease $\cdot$ Rubella

\section{Introduction}

Infectious diseases have been serious risk factors in human societies for centuries. Smallpox has been recorded in human history since more than B.C 1100. People have also been suffering from many other infectious diseases such as malaria, cholera, tuberculosis, typhus, AIDS, influenza, etc. Although people have tried to prevent and hopefully eradicate them, a risk of unknown infectious diseases including SARS, a new type of infectious diseases, as well as Ebola haemorrhagic fever and Zika fever have appeared on the scene.

A model of infectious disease has been studied for years. SIR (Susceptible, Infected, Recovered) model has been widely used to analyse such diseases based

\footnotetext{
S. Kurahashi (凶)

University of Tsukuba, Graduate School of Business Sciences,

3-29-1 Otsuka, Bunkyo, Tokyo, Japan

e-mail: kurahashi.setsuya.gf@u.tsukuba.ac.jp 
on a mathematical model. After an outbreak of SARS, the first SIR model of SARS was published and many researchers studied the epidemic of the disease using this model. When an outbreak of a new type of influenza is first reported, the U.S. government immediately starts an emergency action plan to estimate parameters of its SIR model. Nevertheless, the SIR model has difficulty to analyse which measures are effective because the model has only one parameter to represent infectiveness. For example, it is difficult for the SIR model to evaluate the effect of temporary closing of classes because of the influenza epidemic. The agent-based approach or the individual-based approach has been adopted to conquer these problems in recent years [1-4]. The model enables to represent behaviour of each person. It also reveals the spread of an infection by simulation of the contact process among people in the model.

In this study, we developed a model to simulate rubella based on the infectious disease studies using agent-based modelling. What we want to know is how to prevent an epidemic of infectious diseases not only using mechanisms of the epidemic but also decision-making of health policy [5]. We aim to study the relationship between antibody holding rate of men and the spread of infection by constructing infection of rubella virus with the agent-based model and repeating simulation experiment on a computer. Although our previous study described the infectious disease model of smallpox and Ebola [6], this paper proposes a new model of rubella which has caused crucial problems for pregnant women in recent years. In Sect. 2, as examples of infections that occurred in the past, we will explain smallpox, Ebola hemorrhagic fever, Zika fever and rubella. Section 3 describes related research on infectious disease models. Section 4 explains the basic model and describes the rubella model. Section 5 explains the experimental results and Sect. 6 discusses them. Finally, we summarize the whole in Sect. 7.

\section{Cases of Infectious Disease}

\subsection{Smallpox}

The smallpox virus affects the throat where it invades into the blood and hides in the body for about 12 days. Patients developed a high fever after that, but rashes do not appear until about 15 days after the infection. While not developing rashes, smallpox virus is able to infect others. After 15 days, red rashes break out on the face, arms and legs, and subsequently they spread over the entire body. When all rashes generate pus, patients suffer great pains; finally, $30 \%$ of patients succumb to the disease. For thousands of years, smallpox was a deadly disease that resulted in thousands of deaths. 


\subsection{Ebola Haemorrhagic Fever}

A source of Ebola infection is allegedly by eating a bat or a monkey, but it is unknown whether eating these animals is a source of the infection. Due to the recent epidemic, which began in Guinea in December 2013, 11,310 deaths have been confirmed. The authorities of Guinea, Liberia and Sierra Leone have each launched a state committee of emergency and have taken measures to cope with the situation. The prohibition of entry over the boundary of Guinea is included in these measures.

\subsection{Zika Fever}

Zika fever is an illness caused by Zika virus via the bite of mosquitoes. It can also be potentially spread by sex according to recent report [7, 8]. Most cases have no symptoms and present are usually mild including fever, red eyes, joint pain and a rash [9], but it is believed that the Zika fever may cause microcephaly which severely affects babies by a small head circumference.

\subsection{Rubella}

Rubella is a type of viral infection caused by the rubella virus [10, 11]. In Japan, there were epidemics $(1976,1982,1987,1992)$ once every 5 years, but after a male and female infant was the subject of periodic vaccination, no big epidemic occurred. However, in 2004, 40,000 people outbreaks were estimated and ten congenital rubella syndromes were reported. A large epidemic occurred in Asia in 2011, and from 2013 to 2014 , an epidemic exceeding 14,000 cases occurred mainly in adult males who did not take the vaccine [12]. The epidemic recurred in 2018, as of October the number of annual infections of rubella was about 1300 people, the National Institute of Infectious Diseases announced emergency information on rubella epidemics. The Centers for Disease Control and Prevention (U.S.) raised the rubella alert level in Japan to the second 'recommendation' among the three levels [13]. They recommended that pregnant women who are not protected against rubella through either vaccination or previous rubella infection should not travel to Japan during this outbreak.

\section{Related Work}

\subsection{Smallpox and Bioterrorism Simulation}

Epstein [14, 15] made a smallpox model based on 49 epidemics in Europe from 1950 to 1971 . In the model, 100 families from two towns were surveyed. The family includes two parents and two children, thus the population is each 400 from each 
town. All parents go to work in their town during the day except $10 \%$ of adults who go to another town. All children attend school. There is a communal hospital serving the two towns in which each five people from each town work. This model was designed as an agent-based model, and then simulation of infectious disease was conducted using the model. As results of experiments showed that (1) in a base model in which any infectious disease measures were not taken, the epidemic spread within 82 days and $30 \%$ of people died, (2) a trace vaccination measure was effective but it was difficult to trace all contacts to patients in an underground railway or an airport, (3) a mass vaccination measure was effective, but the number of vaccinations would be huge so it was not realistic and (4) epidemic quenching was also effective, and reactive household trace vaccination along with pre-emptive vaccination of hospital workers showed a dramatic effect.

\subsection{Individual-Based Model for Infectious Diseases}

Ohkusa [16] evaluated smallpox measures using an individual-based model of infectious diseases. The model supposed a town including 10,000 habitats and a public health centre. In the model, one person was infected with smallpox virus at a shopping mall. They compared between a trace vaccination measure and a mass vaccination measure. As a result of simulation, it was found that the effect of trace vaccination dropped if the early stage of infection was high and the number of medical staff is small, while the effect of mass vaccination was stable. Therefore, timely and concentrate mass vaccination is required when virus starts spreading. The estimation about the number, place and time of infection is needed quickly and the preparation of an emergency medical treatment and estimation system is required for such occasions. Regarding measles epidemics, agent-based simulation models of measles transmission have been developed using the Framework for Reconstructing Epidemiological Dynamics, a data-driven agent-based model to simulate the spread of an airborne infectious disease in an Irish town, and so on [17, 18].

Summary of related work From these studies, the effectiveness of an agent-based model has been revealed, yet these are not sufficient models to consider a relationship between antibody holding rate of men and women, and commutation routes and the gender-specific workplace. In addition, authorities need to make a decision regarding measles-rubella mixed (MR) vaccine to men. This study takes into account these extensions.

\section{A Health Policy Simulation Model of Infectious Disease}

We designed a health policy simulation model of infectious disease based on Epstein's smallpox model. The model includes smallpox, Ebola haemorrhagic fever and rubella. 


\subsection{A Base Model of Smallpox}

We assume all individuals to be susceptible which means no background of immunity. 100 families live in two towns.

The family includes two parents and two children. Therefore, the population is each 400 in each town. All parents go to work in their town during the day except $10 \%$ of adults commute to another town. All children attend school. There is a communal hospital serving two towns in which five people from each town work. Each round consists of an interaction through the entire agent population. The call order is randomized each round and agents are processed or activated, serially. On each round, when an agent is activated, she identifies her immediate neighbours for interaction. Each interaction results in a contact. In turn, that contact results in a transmission of the infection from the contacted agent to the active agent with probability.

The probability of contact at an interaction is 0.3 at a workplace and a school, while 1.0 at a home and a hospital. The probability of infection at a contact is 0.3 at a workplace and a school, while 1.0 at a home and a hospital. In the event the active agent contracts the disease, she turns blue to green and her own internal clock of disease progression begins. After 12 days, she will turn yellow and begins infecting others. Length of noncontagious period is 12 days, and early rash contagious period is 3 days. Unless the infected individual is vaccinated within 4 days of exposure, the vaccine is ineffective. At the end of day 15, smallpox rash is finally evident. Next day, individuals are assumed to hospitalize. After 8 more days, during which they have a cumulative $30 \%$ probability of mortality, surviving individuals recover and return to circulation permanently immune to further infection. Dead individuals are coloured black and placed in the morgue. Immune individuals are coloured white. Individuals are assumed to be twice as infectious during days 1-19 as during days 12-15.

\subsection{A Model of Ebola Hemorrhagic Fever}

In the event the active agent contracts the disease, she turns blue to green and her own internal clock of disease progression begins. After 7 days, she will turn yellow and begins infecting others. However, her disease is not specified in this stage. After 3 days, she begins to have vomiting and diarrhoea and the disease is specified as Ebola. Unless the infected individual is dosed with antiviral medicine within 3 days of exposure, the medicine is ineffective. This is an imaginary medicine to play the policy game. At the end of day 12, individuals are assumed to hospitalize. After 4 more days, during which they have a cumulative $90 \%$ probability of mortality, surviving individuals recover and return to circulation permanently immune to further infection. Dead individuals are coloured black and placed in the morgue. Immune individuals are coloured white. Other settings are the same as smallpox. 


\subsection{A Model of Rubella}

Rubella is a viral infectious disease characterized by fever and rash. Since symptoms range from subclinical to complications, it is difficult to judge as rubella only with symptoms. If a pregnant woman until about 20 weeks of pregnancy infects rubella with a virus, there is a possibility that the baby will develop congenital rubella syndrome (CRS). In consequence, congenital heart disease, hearing loss, cataract, pigmentary retinopathy and the like occur as congenital abnormalities.

For this reason, the pre-inoculation of the vaccine is extremely important. In Japan, however, only junior high school girls were eligible for regular vaccination of rubella vaccine from 1977 to 1995 . In the past, vaccination was recommended for children under the age of 3 , but due to the frequent occurrence of meningitis caused by the vaccine strains, the use was discontinued after that. Thereafter, the national epidemic of measles occurred mainly in the 10-20 generations in 2007. 'Prevention guideline on specific infectious diseases related to measles' was announced by the Ministry of Health, Labour and Welfare, and rubella was also designated as a disease to take measures. And during the 5 years from 2008 to 2012, as a periodical inoculation at the first period (1-year-old child), the second period (before elementary school entrance), the third period (first grader of junior high school) and the fourth period (third grader of high school), MR vaccine was to be inoculated. From fiscal 2013, as a rule, measles-rubella mixed (MR) vaccine is inoculated in infants of the first period and children before elementary school entrance of the second period. According to a survey of 2016, females possess about $95 \%$ of antibodies in all ages, while males only possess about $90 \%$ of $20-34$ years old, and 76-84\% of 35-55 years old. The antibodies holding rate in middle-aged males stays low.

Regarding the infection process, fever, rashes and lymphadenopathy (especially the posterior portion of the auricle, the back of the auricle, the neck) appear after a latency period of 14-21 days from infection. Fever is observed in about half of rubella patients. In addition, inapparent infection is about $15-30 \%$.

\section{Experimental Results}

\subsection{A Basic Model of Rubella}

The process of infection in a rubella model is plotted in Fig. 1. The model employs with the basic parameters to the disease. An orange line and a blue line indicate the number of infected and recovered people, respectively. When a player adopts a basic model, it takes approximately 150 days until convergence of the outbreak and more than 29 people have infected.

However, the result of executing the experiment many times is greatly different. Figure 2 shows the histogram of the results of 1000 runs. The horizontal axis shows the number of infected people, the vertical axis shows occurrence frequency, the blue 

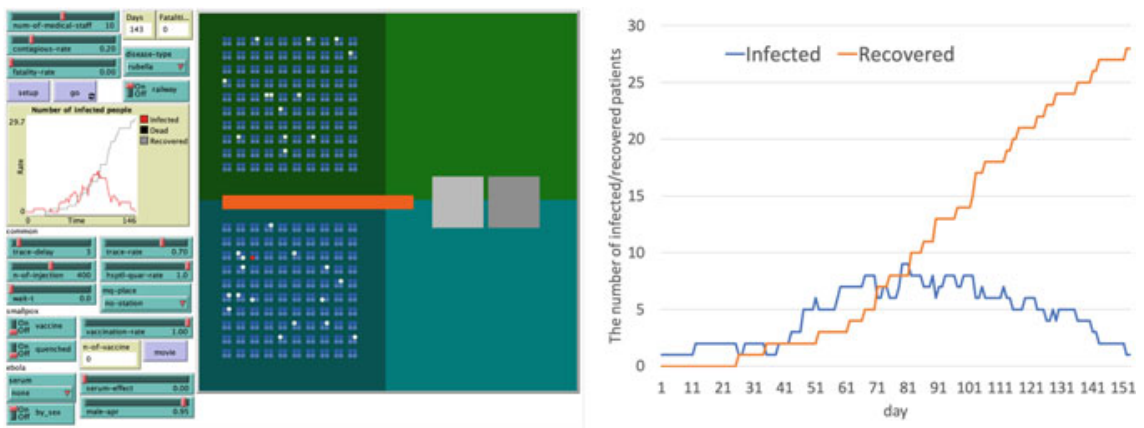

Fig. 1 The simulation model and the experimental result of the rubella basic model

Fig. 2 The frequency chart of the rubella basis model

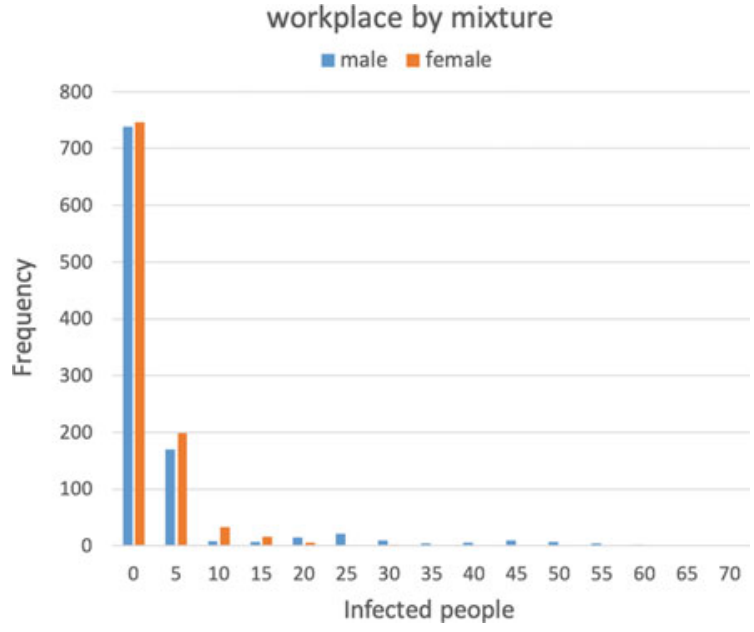

line shows male and the orange line shows female. Infections do not spread in the cases of about $75 \%$, and the number of infected people is about five for males and females in the cases of about $20 \%$. As a result of the experiment, in most cases, it is found that infection has not spread.

\subsection{A Model of the Workplace Separated the Sexes}

Next, the results when men and women are working separately in the workplace are shown in Fig. 3. As the results show, the frequency of infection by more than 20 men has increased to over $10 \%$ of the total. This result is thought to be caused by a low antibody holding rate of males. However, the total number of women infected has not increased. 
Fig. 3 The experimental result of rubella model of the workplace separated the sexes

Fig. 4 The experimental result of rubella model with a railway
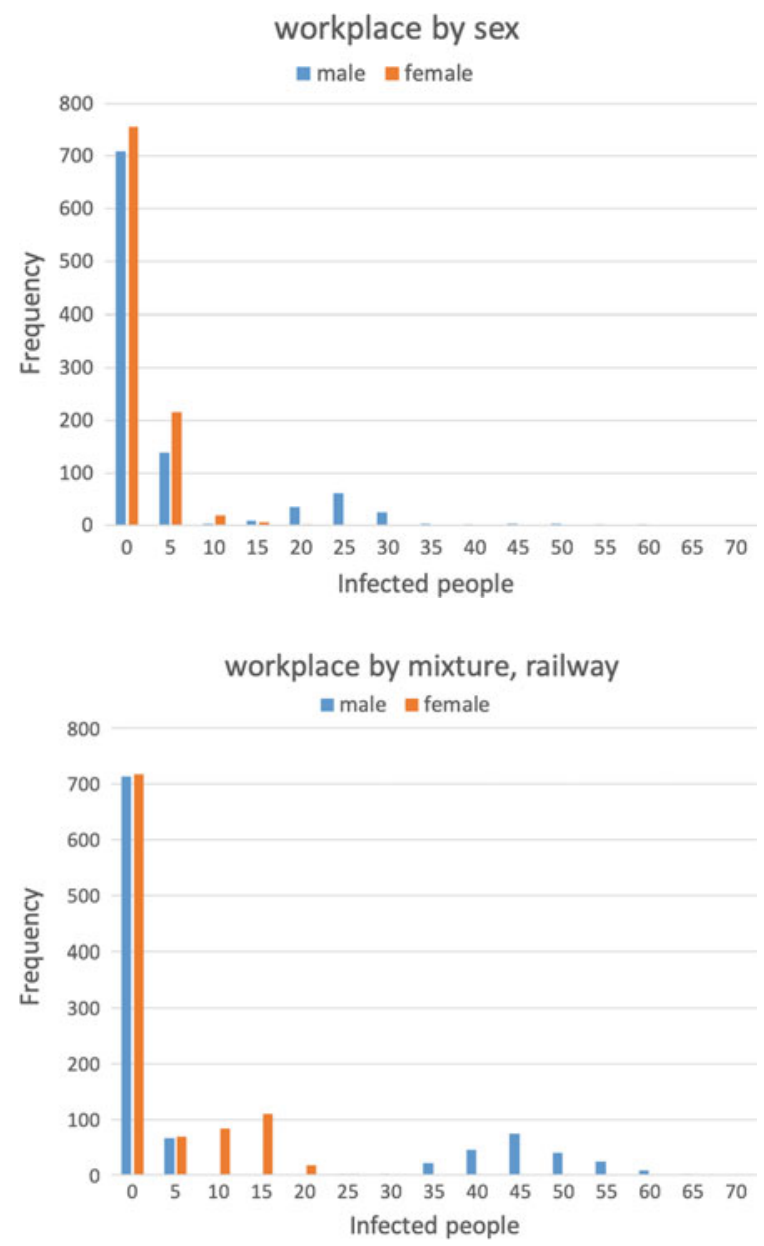

\subsection{A Model with a Railway}

Next, we conducted an experiment with a model that introduced the railway to commute. In this model, adults commute by railway. As the experimental result in Fig. 4 shows, the number of infected men has not only increased dramatically but also the number of women infected has increased.

In the basic model without the railway, the frequency of infection of one or more women became $25 \%$, but in the model using the railway, it has increased to $28 \%$. Especially, the case where more than ten women were infected was $21 \%$ or more, which was a severe result. Figure 5 shows the experimental results when men and women work separately in a railway model. The result is more serious. The frequency 
Fig. 5 The experimental result of rubella model of the workplace separated the sexes with a railway

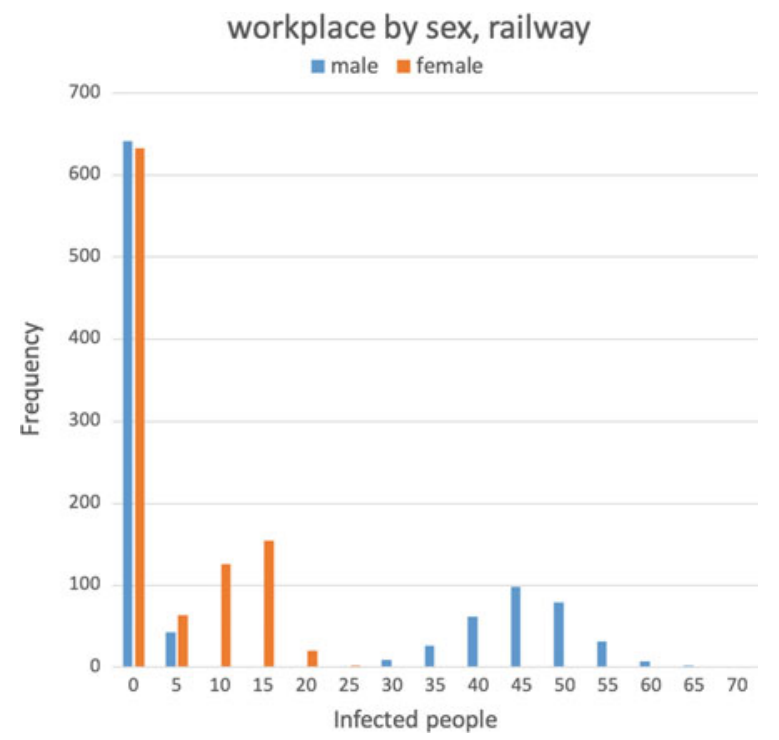

of infection of one or more women increases to $37 \%$, and the case where more than ten women were infected was $30 \%$.

\subsection{A Model of Vaccination to Men in Order to Prevent Infection Spread}

It was estimated by the gender-specific workplace model that the low infection rate of middle-aged men as low as $80 \%$ is the cause of infection spread. Based on this, we experimented in the case of strengthening medical policy promoting vaccination for males and raising the antibody holding rate to $95 \%$.

Figure 6 shows experimental results at the gender-specific workplace, railway use and male antibody holding rate of $95 \%$. According to this, both males and females, the proportion of infected people who expanded to more than one person has drastically decreased to less than $15 \%$. By combining other infection prevention measures, it is possible to control the spread of infection of rubella.

\section{Discussion}

From these experimental results, the rubella virus infection is not represented by a simple statistical distribution. It is considered that the infection process is a complicated system in which positive feedback works by accidentality of infection route 
Fig. 6 The experimental result of rubella model of the workplace separated the sexes with a railway and $95 \%$ antibody holding rate

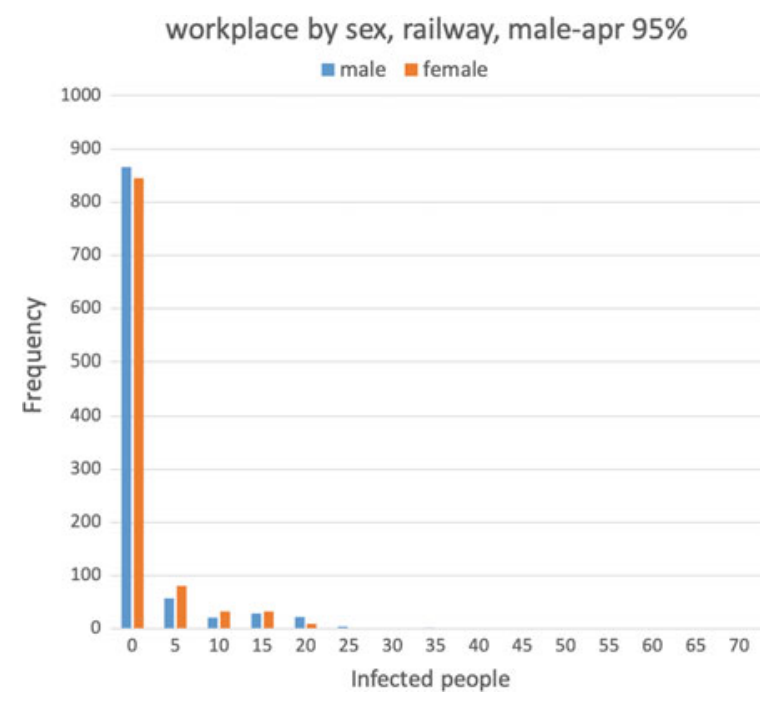

and interaction between infected people. It is also speculated that the spread of infection will start in a workplace where many men do not possess antibodies. One of the major factors of infection spread is commuters with many opportunities to come into close contact with the unspecified majority in a train. In addition, it became clear that raising the antibody holding rate of males is an important measure to prevent the spread of the whole infection.

\section{Conclusion}

This study proposes a simulation model of rubella. It also evaluates health policies to prevent an epidemic. As health policies, vaccination to men, avoidance of separated workplace by sexes and avoidance crowds in a train were implemented in the model. As a result of experiments, it has been found that vaccination to middle-aged men and avoidance crowds in a train are crucial factors to prevent the spread of rubella. Using a public transportation to commute, however, is inevitable in a modern society. Even if $95 \%$ of people including men and women were vaccinated, it would not prevent the epidemic completely. By combining other infection prevention measures, it is possible to control the spread of infection of rubella. 


\section{References}

1. Burke, D.S., et al.: Individual-based computational modeling of smallpox epidemic control strategies. Acad. Emerg. Med. 13(11), 1142-1149 (2006)

2. Longini Jr., I.M., et al.: Containing a large bioterrorist smallpox attack: a computer simulation approach. Int. J. Infect. Dis. 11(2), 98-108 (2007)

3. Gilbert, N.: Agent-Based Models, vol. 153. SAGE, Thousand Oaks (2008)

4. Easley, D., Kleinberg, J.: Networks, crowds, and markets: reasoning about a highly connected world. Cambridge University Press, Cambridge (2010)

5. Okabe, N.: Risk and benefit of immunisation: infectious disease prevention with immunization. Iryo to Shakai 21(1), 33-40 (2011)

6. Kurahashi, S.: A health policy simulation model of smallpox and Ebola haemorrhagic fever. In: 9th International KES Conference on Agents and Multi-agent Systems: Technologies and Applications (KES-AMSTA-15), ams15-030 (2015)

7. Chen, L.H., Hamer, D.H.: Zika virus: rapid spread in the Western Hemisphere. Ann. Intern. Med. (2016)

8. Hennessey, M., Fischer, M., Staples, J.E.: Zika virus spreads to new areas - region of the Americas, May 2015-January 2016. Cent. Dis. Control Prev. 65(3), 55-58 (2016)

9. WHO: Zika virus, World Health Organization, Media centre, Fact sheets, January (2016)

10. National Institute of Infectious Diseases (NIID): About rubella (2018). https://www.niid.go. $\mathrm{jp} / \mathrm{niid} / \mathrm{ja} / \mathrm{kansennohanashi/430-rubella-intro.html}$

11. Centers for Disease Control and Prevention (CDC): Rubella (German Measles, Three-Day Measles) (2018). https://www.cdc.gov/rubella/about/transmission.html

12. National Institute of Infectious Diseases: Fiscal year 2017 rubella immunization status and status of antibody retention - survey on infectious disease epidemic survey in 2017 (provisional result). IASR 39(3), 39-41 (2018)

13. Centers for Disease Control and Prevention (CDC): Rubella in Japan. https://wwwnc.cdc.gov/ travel/notices/alert/rubella-japan

14. Epstein, J.M., et al.: Toward a Containment Strategy for Smallpox Bioterror: An IndividualBased Computational Approach (2002)

15. Epstein, J.M.: Generative Social Science: Studies in Agent-Based Computational Modeling. Princeton University Press, Princeton (2006)

16. Ohkusa, Y.: An evaluation of counter measures for smallpox outbreak using an individual based model and taking into consideration the limitation of human resources of public health workers. Iryo to Shakai 16(3), 284-295 (2007)

17. Liu, F., Enanoria, W.T.A., Zipprich, J., Blumber, S., Harriman, K., Ackley, S.F., Wheaton, W.D., Allpress, J.L., Porco, T.C.: The role of vaccination coverage, individual behaviors, and the public health response in the control of measles epidemics: an agent-based simulation for California. BMC Public Health 15, 447 (2015)

18. Hunter, E., Namee, B.M., Kelleher, J.: An open-data-driven agent-based model to simulate infectious disease outbreaks, PLOS ONE 14(1), e0211245 (2019). https://doi.org/10.1371/ journal.pone. 0211245 